\title{
Hemolytic Uremic Syndrome Caused by Mycoplasma Pneumoniae Infection in Children: A Case Report and Literature Review
}

\author{
Sirinthip Kittivisuit, M.D., Prayong Vachvanichsanong, M.D., Thirachit Chotsampancharoen, M.D.
}

Department of Pediatrics, Faculty of Medicine, Prince of Songkla University, Hat Yai, Songkhla 90110, Thailand.

Received 29 September 2021 • Revised 10 October 2021 • Accepted 11 October 2021 • Published online 26 October 2021

\begin{abstract}
:
We describe the case of a 6-year-old boy with a Mycoplasma pneumoniae (M. pneumoniae) respiratory tract infection associated with thrombotic microangiopathic hemolytic anemia and thrombocytopenia with renal failure which was diagnosed as atypical hemolytic uremic syndrome. Renal biopsy showed features of thrombotic microangiopathy. The patient was treated with azithromycin for the $M$. pneumoniae infection, and supportive care with red cell transfusion and renal dialysis in the acute period. The microangiopathic hemolytic anemia and thrombocytopenia resolved within 2 months after diagnosis but the renal function damage was irreversible. The patient developed end-stage renal disease and required long term renal replacement therapy.
\end{abstract}

Keywords: end-stage renal disease, hemolytic uremic syndrome, mycoplasma pneumoniae, thrombotic microangiopathy

\section{Introduction}

Hemolytic uremic syndrome (HUS) is characterized by the combination of microangiopathic hemolytic anemia with thrombocytopenia and renal failure. ${ }^{1}$ The annual incidence of HUS in children ranges from 7.1 to 14.2 per million. It can occur in any age group but most cases are reported in children under 5 years of age. ${ }^{2-4}$ HUS in childhood has various etiologies but the most common cause is from an infectious origin such as Shiga toxin-producing Escherichia coli or Shigella dysenteriae type 1, either of which cause a disease known as typical or diarrheal-associated HUS. Other infectious causes are called atypical HUS or nondiarrheal-associated HUS, which has been reported as arising from various organisms including both viruses and bacteria. ${ }^{1}$ Mycoplasma pneumoniae (M. pneumoniae) is one of the pathogens which very rarely causes HUS. Usually, $M$.
Contact: Thirachit Chotsampancharoen, M.D.

Department of Pediatrics, Faculty of Medicine, Prince of Songkla University, Hat Yai, Songkhla 90110, Thailand.

E-mail: cthirachit@yahoo.com, cteerach@medicine.psu.ac.th

(c) 2021 JHSMR. Hosting by Prince of Songkla University. All rights reserved.

This is an open access article under the CC BY-NC-ND license

(http://www.jhsmr.org/index.php/jhsmr/about/editorialPolicies\#openAccessPolicy).
J Health Sci Med Res 2022;40(4):475-480 doi: 10.31584 /jhsmr.2021848 www.jhsmr.org 
pneumoniae are manifestations of a pulmonary infection, but atypical extrapulmonary clinical manifestations have been reported. ${ }^{5}$ Herein, we report the case of an extrapulmonary manifestation of M. pneumoniae associated HUS.

\section{Case report}

A previously healthy 6-year-old boy was transferred to our hospital with chief complaints of anemia and bleeding diathesis, following fever and productive cough for 5 days before these symptoms developed. He was oliguric. There was no family history of hematological disorders. His vital signs showed a temperature of 39 degrees Celsius, blood pressure 120/84 millimeters of mercury, heart rate 140 beats per minute, and respiratory rate 50 breaths per minute. The physical examination was unremarkable except for pallor and multiple ecchymoses at the trunk. The blood tests showed a hemoglobin level of 8.5 grams per deciliter $(\mathrm{g} /$ $\mathrm{dL})$, reticulocyte count of $18.0 \%$, platelet count of 29,000 per cubic millimeter $\left(\mathrm{mm}^{3}\right)$, and normal white blood cell count. Impaired renal function was found, with blood urea nitrogen 58.0 milligrams per deciliter $(\mathrm{mg} / \mathrm{dL})$ and serum creatinine $2.9 \mathrm{mg} / \mathrm{dL}$. Hemolytic markers showed a raised lactate dehydrogenase level of 6,706 units per liter (U/) and reticulocytosis. A peripheral blood smear showed $5.0 \%$ schistocytes, which was compatible with microangiopathic hemolytic anemia (MAHA). Coagulation tests were normal, including fibrin degradation products and D-dimer. Direct and indirect Coombs and cold agglutinin tests were negative. Urinalysis revealed numerous red blood cells and proteinuria with a urine protein-to-creatinine ratio of $150 \mathrm{mg} / \mathrm{mg}$. A chest radiograph was normal. Renal ultrasonography showed normal size for age, no hydronephrosis and nonspecific increased cortical echogenicity. A diagnosis of thrombotic microangiopathy (TMA) was made and diagnostic workups of HUS and thrombotic thrombocytopenic purpura (TTP) were undertaken according to the European guidelines. ${ }^{6} \mathrm{~A}$ stool culture was negative for enterohemorrhagic E. coli. Hemocultures and urine culture were negative for Streptococcus pneumoniae. A disintegrin and metalloproteinase with a thrombospondin type 1 motif, membrane 13 (ADAMTS13) activity test was $71.0 \%$ (normal range 58.0$170.0 \%$ ). The serum C3 complement was $41 \mathrm{mg} / \mathrm{dL}$ (normal range $90-180 \mathrm{mg} / \mathrm{dL}$ ). The antinuclear antibodies and antidouble-stranded deoxyribonucleic acid antibodies were negative, and the anti-streptolysin O titer was <200 international unit (IU). An initial serum titer to detect $M$. pneumoniae by gel particle agglutination antibody assay (Fujirebio, Japan) was performed, which was 1:80; a second titer 2 weeks later was 1:320. This second titer gave a definite diagnosis of $M$. pneumoniae because of the 4-fold increase in the titer. A renal biopsy was performed 2 weeks after admission and the renal pathology confirmed the diagnosis with TMA (Figure 1). Immunofluorescent staining showed strong endothelial C3 depositions in the glomeruli and negative staining for a C1q complement. Electron microscopy (EM) showed endothelial cell swelling and stagnant red blood cells. Overall, the renal pathology was definite for a diagnosis of HUS and serology confirmed M. pneumoniae infection. The patient was treated with azithromycin for M. pneumoniae infection, and supportive care with red cell transfusion. The renal dialysis was performed in the acute period, but the patient developed irreversible renal function requiring long-term renal replacement therapy. Eculizumab was not used because it was unavailable in our center. The platelet counts steadily increased, exceeding 150,000 per $\mathrm{mm}^{3}$ on day 42 . The hemoglobin level gradually normalized over 60 days without red blood cell transfusion. The serum C3 complement assay was repeated and was normal 6 months after the initial diagnosis. The patient had permanent kidney damage and 24 months after contracting the disease still needed renal replacement therapy, and it was believed the damage was irreversible. 



Figure 1 Histopathology. A: Fibrinoid necrosis in blood vessel $(\mathrm{T})$ and endothelial cell swelling (periodic acid-Schiff stain). B: Microthrombi in the capillary lumens of glomerulus and collapsed glomerulus (hematoxylin and eosin stain). C: Microthrombi in the capillary lumens of glomerulus (Masson-Trichrome stain). D: Thickening of the glomerular basement membrane (periodic acid sliver-methenamine stain).

\section{Discussion}

M. pneumoniae associated HUS has been reported in 4 cases in children and 1 case in an adult since 1976 as shown in Table 1. ${ }^{7-11} M$. pneumoniae was mentioned in the first two cases of HUS, but could not be confirmed as $M$. pneumoniae infection in the first case, a patient with cold agglutinin disease ${ }^{7}$, while the second case was diagnosed as $M$. pneumoniae caused hemolytic anemia associated with anti-P autoantibody. ${ }^{8}$ Only the most recent three cases had confirmed M. pneumoniae complicated with HUS. ${ }^{9-11}$

HUS is a microvascular occlusion disorder in the TMA category. TMA is defined by histological lesions found in the arterioles and capillaries and characterized by thickening of the vascular walls, especially endothelial swelling and detachment, and subendothelial accumulation of cell debris. In patients with TMA, formation of fibrin and platelet-rich thrombi occur in the microcirculation, obstructing the vessel lumen leading to end-organ ischemia and infarction. HUS is diagnosed by clinical signs characterized by TMA and renal involvement. ${ }^{1}$ TMA on renal pathology has been reported in 3 cases of HUS associated with post-streptococcal glomerulonephritis. ${ }^{12}$ However, to date TMA has not been demonstrated in the renal pathology of M. pneumoniae complicated with HUS in children. There is one case report in an adult with acute kidney injury and hemolytic anemia secondary to $M$. pneumoniae infection 





whose renal pathology showed features of postinfectious glomerulonephritis, but the glomerular microangiopathic injury was detected only by EM, not light microscopy. ${ }^{11}$ Usually a renal biopsy is not indicated for diagnosis but our case had one performed which also supported the clinical diagnosis of HUS, and ours may be the first case with demonstrated evidence of TMA on renal pathology in M. pneumoniae complicated with HUS in a child.

Autoimmune hemolytic anemia can be a severe complication of a $M$. pneumoniae infection and should thus be included in the differential diagnosis of HUS. The mechanism of hemolysis has been postulated as involving the mechanism of cold agglutin autoantibodies against red blood cells. ${ }^{5}$ In addition, our patient had the clinical combination of MAHA, thrombocytopenia, nephrotic range proteinuria with hematuria and hypertension with negative cold agglutinin, and a Coombs test led us to rule out autoimmune hemolytic anemia. Disseminated intravascular coagulation was ruled out by normal coagulation tests. Our case had serological confirmation by a 4-fold rise in the $M$. pneumoniae antibodies.

Atypical HUS is a chronic condition and involves a poorer prognosis than typical HUS. ${ }^{13,14}$ Studies reported that half of atypical HUS children needed dialysis at admission, and $40.0-60.0 \%$ of patients never regained renal function, as in our patient. ${ }^{13,14}$ After the first episode, mortality has been reported to be higher in children than in adults, but progression to end-stage renal disease (ESRD) is more frequent in adults. The prognosis of atypical HUS also depends on the patient's genetic background ${ }^{13,14}$, and the lack of genetic screening of the alternative complement pathway may be a limitation of our case.

Eculizumab, a monoclonal anti-C5 antibody, is the first-line therapy for atypical HUS and accounts for better outcomes, especially in the prevention of ESRD. Unfortunately, eculizumab was not used in our case because it was unavailable in our center, and our case developed ESRD which required long term renal replacement therapy. However, there are reports of previous cases of M. pneumoniae associated with HUS in children who recovered renal function after receiving supportive care without eculizumab treatment. Godron et al. ${ }^{9}$ reported a 1-year-old child with M. pneumoniae complicated with HUS with a good prognosis, in a patient who had only very mild renal impairment and recovered very well with minimal treatment. Bugajer-Gleitman et al. ${ }^{7}$ and Miklaszewka et al. ${ }^{10}$ reported two cases which required short-term peritoneal dialysis in the acute period with good clinical response.

The most common hypothesis in the literature for an infectious trigger of atypical HUS is alternative complement pathway dysregulation. ${ }^{1}$ Our patient had transient low serum C3 during the event and immunofluorescent staining showed strong C3 complement deposited on the mesangial cells and the serum C3 complement returned to normal 6 months after the disease was first diagnosed. If we were to propose a hypothesis to explain the cause of symptoms in our patient, the hypothesis would be transient activation of the alternative complement pathway induced by $M$. pneumoniae itself as documented in Shiga toxinproducing $E$. coli and $S$. pneumoniae. ${ }^{1}$ The identification of a previous case of acute postinfectious glomerulonephritis with transient hypocomplementemia (low serum C3 complement) associated with $M$. pneumoniae suggests transient complement activation. ${ }^{15}$ The excessive activation of the alternative complement pathway results in endothelial cell injury and accumulation of the proinflammatory and procoagulant components that eventually trigger thrombosis. ${ }^{1}$

\section{Conclusion}

In conclusion, we report an unusual case of a child with $M$. pneumoniae infection associated with HUS, leading to the suggestion that $M$. pneumoniae should be in the differential diagnosis of atypical HUS in cases with 
a recent history of respiratory symptoms. M. pneumoniae associated HUS in children has been reported to result in a good renal outcome with supportive care. But in some situations, no eculizumab treatment may lead to irreversible renal damage, as in our case.

\section{Acknowledgement}

We would like to thank Dave Patterson of the Office of International Affairs of the Faculty of Medicine, Prince of Songkla University for manuscript editing.

\section{Conflict of interest}

None to declare

\section{References}

1. Fakhouri F, Zuber J, Frémeaux-Bacchi V, Loirat C. Haemolytic uraemic syndrome. Lancet 2017;390:681-96.

2. Lynn RM, O’Brien SJ, Taylor CM, Adak GK, Chart H, Cheasty T, et al. Childhood hemolytic uremic syndrome, United Kingdom and Ireland. Emerg Infect Dis 2005;11:590-6.

3. Espié E, Grimont F, Mariani-Kurkdjian P, Bouvet P, Haeghebaert S, Filliol I, et al. Surveillance of hemolytic uremic syndrome in children less than 15 years of age, a system to monitor 0157 and non-O157 Shiga toxin-producing Escherichia coli infections in France, 1996-2006. Pediatr Infect Dis J 2008;27:595-601.

4. Schifferli A, von Vigier RO, Fontana M, Spartà G, Schmid $H$, Bianchetti MG, et al. Hemolytic-uremic syndrome in Switzerland: a nationwide surveillance 1997-2003. Eur J Pediatr 2010;169: 591-8.

5. Narita M. Classification of extrapulmonary manifestations due to Mycoplasma pneumoniae infection on the basis of possible pathogenesis. Front Microbiol 2016;7:23.

6. Ariceta G, Besbas N, Johnson S, Karpman D, Landau D, Licht $\mathrm{C}$, et al. Guideline for the investigation and initial therapy of diarrhea-negative hemolytic uremic syndrome. Pediatr Nephrol 2009;24:687-96.

7. Bugajer-Gleitman HE, Balzar E, Lubec G, May L, Weissenbacher $G$. The presence of cold agglutinins in hemolytic uremic syndrome. Padiatr Padol 1976;11:245-53.

8. Fitzpatrick MM, Walters MD, Trompeter RS, Dillon MJ, Barratt TM. Atypical (non-diarrhea-associated) hemolytic-uremic syndrome in childhood. J Pediatr 1993;122:532-7.

9. Godron A, Pereyre S, Monet C, Llanas B, Harambat J. Hemolytic uremic syndrome complicating Mycoplasma pneumoniae infection. Pediatr Nephrol 2013;28:2057-60.

10. Miklaszewska M, Zachwieja K, Drożdż D, Pállinger É, Takács B, Szilágyi Á, et al. Hemolytic uremic syndrome with Mycoplasma pneumoniae infection and membrane cofactor protein mutation - case report. Przegl Lek 2016;73:862-4.

11. Carrara C, Abbate M, Sabadini E, Remuzzi G. Acute kidney injury and hemolytic anemia secondary to Mycoplasma pneumoniae infection. Nephron 2017;137:148-54.

12. Kakajiwala A, Bhatti T, Kaplan BS, Ruebner RL, Copelovitch L. Post-streptococcal glomerulonephritis associated with atypical hemolytic uremic syndrome: to treat or not to treat with eculizumab? Clin Kidney J 2016;9:90-6.

13. Noris M, Caprioli J, Bresin E, Mossali C, Pianetti G, Gamba $\mathrm{S}$, et al. Relative role of genetic complement abnormalities in sporadic and familial aHUS and their impact on clinical phenotype. Clin J Am Soc Nephrol 2010;5:1844-59.

14. Fremeaux-Bacchi V, Fakhouri F, Garnier A, Bienaimé F, Dragon-Durey MA, Ngo S, et al. Genetics and outcome of atypical hemolytic uremic syndrome: a nationwide French series comparing children and adults. Clin J Am Soc Nephrol 2013;8:554-62.

15. Adra AL, Vigue MG, Dalla Vale F, Ichay L, Raynaud P, Mariani $A$, et al. Favorable outcome in a case of Mycoplasma pneumoniae-associated crescentic glomerulonephritis. Pediatr Nephrol 2010;25:1765-9. 